\title{
Weakly Compact Operators and Interpolation
}

\author{
LECH MALIGRANDA* \\ Departamento de Matemáticas, IVIC Apartado 21827, Caracas 1020A, Venezuela
}

(Received: 27 April 1992)

\begin{abstract}
The class of weakly compact operators is, as well as the class of compact operators, a fundamental operator ideal. They were investigated strongly in the last twenty years. In this survey, we have collected and ordered some of this (partly very new) knowledge. We have also included some comments, remarks and examples.
\end{abstract}

Mathematics Subject Classifications (1991): 47B07, 47A57

Key words: weakly compact operators, Banach space, interpolation

\section{Introduction}

The norm topology is too strong to allow any widely applicable subsequential extraction principles. Indeed, in order that each bounded sequence in $X$ has a norm convergent subsequence, it is necessary and sufficient that $X$ be finite dimensional. This fact leads us to consider another, weaker topology (weak topology) on normed linear spaces which is related to the linear structure of the spaces and to search for subsequential extraction principles therein. First of all, we will discuss the weak topology, weak compactness and reflexivity of Banach spaces. Then we would like to give an exposition of weakly compact operators between Banach spaces and Banach lattices. Almost all of this material is in principle available in some books.

Finally, in $\S 5$ there are considerations on interpolation of weakly compact operators from the recent work of Maligranda and Quevedo [20]. We also give some additional comments and remarks.

\section{Weakly Compact Sets and Reflexivity}

Let $X$ be an infinite-dimensional Banach space. The weak topology $\sigma\left(X, X^{*}\right)$ is the weakest topology for which all bounded linear functionals on $X$ are continuous, i.e., a net $\left(x_{\alpha}\right)$ converges weakly to $x_{0}$ if, for each $x^{*} \in X^{*}$, $\lim _{\alpha} x^{*}\left(x_{\alpha}\right)=x^{*}\left(x_{0}\right)$.

The weak topology is linear (addition and scalar multiplication are continuous), Hausdorff (weak limits are unique) and not metrizable.

* Current address: Department of Mathematics, Lulea University, S-95187 Lulea, Sweden. 
EXAMPLE 2.1 (Neumann) Let $A=\left\{e_{m}+m e_{n}: m<n, m, n \in \mathbf{N}\right\}$ be a set in $\ell_{2}$. Then $0 \in \bar{A}^{\text {weak }}$, but there is no sequence in $A$ which is weakly null. Therefore, the weak topology need not be metrizable.

Ryff [28] also constructed a subset of $\ell_{1}$ which is bounded, weakly sequentially closed and not weakly closed. This example (as well as Neumann's) points out a norm closed set whose weak closure is obtained by adding a single point: the origin.

The weak topology is not complete, but some of the Banach spaces are sequentially weakly complete.

For any subset $A \subset X$ we have $\bar{A}^{\|\|} \subset \bar{A}^{\text {weak }}$.

THEOREM 2.2 (Mazur) If $A$ is a convex subset of a Banach space $X$, then $\bar{A}^{\|} \|=\bar{A}^{\text {weak }}$.

A subset $A \subset X$ is said to be weakly compact if it is compact in the weak topology of $X . A$ is said to be relatively weakly compact if the closure $\bar{A}^{\text {weak }}$ is weakly compact. $A$ is said to be sequentially weakly compact if, for every sequence of elements of $A$, there is a subsequence which is weakly convergent to an element of $A$.

The map $\kappa: X \rightarrow X^{* *}$ defined by $(\kappa x)\left(x^{*}\right)=x^{*}(x)$ for $x \in X, x^{*} \in X^{*}$ is called the canonical embedding of $X$ into $X^{* *}$. A Banach space $X$ is said to be reflexive if $\kappa(X)=X^{* *}$.

Let $A$ be a weakly compact set in $X$ and $x^{*} \in X^{*}$. Then by the BanachSteinhaus theorem $x^{*}$ is weakly continuous. Therefore $x^{*}(A)$ is a compact set of scalars and $x^{*}(A)$ is bounded for each $x^{*} \in X^{*}$, i.e. $A$ is bounded. Further, $A$ is weakly compact, hence weakly closed, and so norm closed. Conclusion: weakly compact sets are norm closed and norm bounded. Fortunately, closed bounded sets need not be weakly compact.

Weakly compact sets in Banach spaces are plainly different from general compact Hausdorff spaces because they are sequentially compact, and each subset of a weakly compact set has a closure that is sequentially determined.

THEOREM 2.3 (Eberlein-Smulian) A subset of a Banach space $X$ is relatively weakly compact if and only if it is relatively weakly sequentially compact. In particular, a subset of a Banach space is weakly compact if and only if it is sequentially weakly compact.

EXAMPLE 2.4 The unit ball of $\ell_{1}$ is not weakly compact because by Schur's theorem in $\ell_{1}$, weak and norm convergence of sequences coincide.

THEOREM 2.5 (Krein-Smulian) If $A$ is a weakly compact subset of a Banach space $X$, then $\overline{\operatorname{conv}}(A)$ is also weakly compact. 
From Theorem 2.3 we have some properties of weakly compact sets and the classical characterization of reflexivity.

THEOREM 2.6 Let $X$ be a Banach space. Then the following are equivalent:

i) $X$ is reflexive;

ii) the unit ball of $X$ is weakly compact;

iii) the unit ball of $X$ is sequentially weakly compact;

iv) $X^{*}$ is reflexive.

Many interesting characterizations of reflexivity have been given by R.C. James. James (1951) supplied also a counter-example showing that the assumption $\kappa(X)=X^{* *}$ cannot be replaced by $X$ to be isometrically isomorphic to $X^{* *}$.

The classical Banach spaces play a central role in the development of general Banach space theory. How are the criteria for weak compactness or what does it mean for a sequence to converge weakly in these spaces? Let us give two examples.

EXAMPLE 2.7 Let $\Omega$ be any compact Hausdorff space. A bounded sequence in the space $C(\Omega)$ is weakly convergent to zero if and only if it converges pointwise to zero.

EXAMPLE 2.8 (Dunford-Pettis) Let $A$ be a bounded subset of $L_{1}(\Omega, \mu)$ with $\mu \Omega<\infty$. A set $A$ is relatively weakly compact if and only if $\lim _{\mu e \rightarrow 0} \sup _{x \in A}$ $\left\|x 1_{e}\right\|_{L_{1}}=0$.

More about weakly compact sets can be found in Lindenstrauss's survey paper [18] and Floret's monograph [13].

\section{Weakly Compact Operators and Factorization}

A bounded linear operator $T: X \rightarrow Y$ between Banach spaces $X$ and $Y$ is weakly compact if $T\left(B_{X}\right)$ is relatively weakly compact. The weakly compact operators were used for the first time by S. Kakutani and K. Yosida in 1938.

Let us give some examples.

EXAMPLE 3.1 If either $X$ or $Y$ is reflexive Banach space then every bounded linear operator $T: X \rightarrow Y$ is weakly compact.

The proof follows from the fact that in a reflexive Banach space any bounded set is relatively weakly compact and from the following Banach-Dunford theorem: A linear map $T: X \rightarrow Y$ between the Banach spaces $X$ and $Y$ is norm-to-norm continuous if and only if $T$ is weak-to-weak continuous. 
EXAMPLE 3.2 Compositions of a weakly compact operator with a bounded operator (or bounded with weakly compact) are weakly compact.

EXAMPLE 3.3 Let $k \in L_{\infty}([0,1] \times[0,1])$ and $1 \leq p<\infty$. Then the operator $K: L_{1}([0,1]) \rightarrow L_{p}([0,1])$ defined by

$$
K x(t)=\int_{0}^{1} k(s, t) x(s) d s
$$

is weakly compact.

Indeed, for $x \in L_{1}$ and a measurable subset $e$ of $[0,1]$,

$$
\left\|(K x) 1_{e}\right\|_{p} \leq(m e)^{\frac{1}{p}} \sup _{s, t \in[0,1]}|k(s, t)|\|x\|_{1} .
$$

Therefore, if $1<p<\infty$, then putting $e=[0,1]$ we have that $K$ is bounded and from the reflexivity of $L_{p}$ it follows that $K$ is weakly compact. If $p=1$, then $\sup _{\|x\|_{1} \leq 1}\|K x\|_{1}<\infty$ and $\lim _{m e \rightarrow 0} \sup _{\|x\|_{1} \leq 1}\left\|(K x) 1_{e}\right\|_{1}=0$, and by the Dunford-Pettis criterion, $K\left(B_{L_{1}}\right)$ is relatively weakly compact in $L_{1}$.

EXAMPLE 3.4 Let, for $1 \leq p<\infty, V_{p}$ be the Banach space of continuous functions on $[0,1]$ with the finite $p$-variation

$$
v_{p}(x)=\sup \left(\sum_{k=1}^{n}\left|x\left(t_{k}\right)-x\left(t_{k-1}\right)\right|^{p}\right)^{\frac{1}{p}}
$$

where the supremum is taken over all partitions $0=t_{0}<t_{1}<\ldots<t_{n}=1$ of $[0,1]$. The norm is defined by

$$
\|x\|_{V_{p}}=\|x\|_{C}+v_{p}(x) \text {. }
$$

The embedding of $V_{p}$ into $C([0,1])$ is not weakly compact. Namely, let $x_{n}(t)=$ $\frac{1}{2}-\frac{n t}{2}$ if $0 \leq t \leq \frac{1}{n}$ and $x_{n}(t)=0$ if $\frac{1}{n} \leq t \leq 1$. Then $\left\|x_{n}\right\|_{V_{p}}=1,\left\{x_{n}\right\}$ is weakly Cauchy in $C([0,1])$ and $\left\{x_{n}\right\}$ does not have a weakly convergent subsequence to a continuous function on $[0,1]$.

Reflexive spaces play a central role for weakly compact operators because of an important factorization theorem. Before stating this theorem, a Davis-FigielJohnson-Pelczynski construction is needed.

Let $X$ be a Banach space and let $W$ be a convex, symmetric, norm bounded subset of $X$. For each $n$ we put $U_{n}=W+2^{-n} B_{X}$ and denote by $j_{n}$ the Minkowski functional of $U_{n}$. For $0<\theta<1$ and $1<p<\infty$ we set

$$
Z=Z_{\theta, p}=\left\{x \in X:\|x\|=\left(\sum_{n \in \mathbf{N}}\left(2^{-\theta n} j_{n}(x)\right)^{p}\right)^{\frac{1}{p}}<\infty\right\},
$$

and let $J: Z \rightarrow X$ denote the natural inclusion. We refer to $Z$ as the space of Davies, Figiel, Johnson, and Pelczynski, or DFJP space for short. 
LEMMA 3.5 (DFJP, [11]) a) $Z$ is a Banach space and $J$ is continuous.

b) $J$ is Tauberian, i.e., $x^{* *} \in X^{* *}, J^{* *} x^{* *} \in Y$ imply $x^{* *} \in X$.

c) $Z$ is reflexive if and only if $W$ is relatively weakly compact.

THEOREM 3.6 (Davis-Figiel-Johnson-Pelczynsky, [11]) Weakly compact operators factor through reflexive spaces, i.e., if $T: X \rightarrow Y$ is a weakly compact operator, then there is a reflexive Banach space $Z$ and bounded linear operators $S: X \rightarrow Z, R: Z \rightarrow Y$ such that $R S=T$.

Proof. Let $T: X \rightarrow Y$ be weakly compact and let $W=T\left(B_{X}\right)$. The operators $J^{-1} \circ T: X \rightarrow Z$ and $J: Z \rightarrow Y$ provide the required factorization. Q.E.D.

The above factorization theorem can be used to prove several standard results from antiquity.

THEOREM 3.7 (Gantmacher) Let $T: X \rightarrow Y$ be a bounded linear operator between Banach spaces. Then the following are equivalent:

i) $T$ is weakly compact;

ii) $T^{* *}\left(X^{* *}\right) \subset \kappa(Y)$;

iii) $T^{*}$ is weakly compact.

\section{Weakly Compact Operators on Banach Lattices}

A Banach lattice $X$ is a Riesz space with a lattice norm. A Banach lattice is said to have order continuous norm whenever $x_{\alpha} \downarrow 0$ implies $\left\|x_{\alpha}\right\| \downarrow 0$. A Banach lattice $X$ is a $K B$-space (Kantorovich-Banach space) whenever every increasing norm bounded sequence of $X^{+}=\{x \in X: x \geq 0\}$ is norm convergent.

A subset $A \subset X$ is said to be solid whenever $|x| \leq|y|$ in $X$ and $y \in A$ imply $x \in A$. The solid hull of a set $A$ is the smallest solid set that contains $A$ and is precisely the set $\operatorname{sol}(A):=\{x \in X: \exists y \in A$ with $|x| \leq|y|\}$.

The main question here is:

QUESTION 1 Does a weakly compact operator between two Banach lattices factor through a reflexive Banach lattice?

Let us note that if in Lemma $3.5 X$ is a Banach lattice and $W$ is also a solid set then the DFJP space $Z$ is a Banach lattice. Therefore the answer to the main question will be 'yes' if $\operatorname{sol}\left(T\left(B_{X}\right)\right)$ is relatively weakly compact.

LEMMA 4.1 a) (Abramovich, [1]) If $X$ is a $K B$-space and $A$ is relatively weakly compact set, then $\operatorname{sol}(A)$ is relatively weakly compact.

b) (Wickstead, [30]) $A$ Banach lattice $X$ has order continuous norm if and only if for every relatively weakly compact subset $A$ of $X^{+} \operatorname{sol}(A)$ is relatively weakly compact. 
EXAMPLE 4.2 (Meyer-Nieberg, [22]) In a Banach lattice with order continuous norm the solid hull of a relatively weakly compact set need not be relatively weakly compact. Let $X=c_{o}\left(L_{1}([0,1])\right)$ and $x_{n}=\left(x_{k}^{n}\right)$ with $x_{k}^{n}=\sin n t$ if $k \leq n$ and $x_{k}^{n}=0$ if $k>n$. Then $\left\{x_{n}: n \in \mathbf{N}\right\}$ is relatively weakly compact and $\left\{\left|x_{n}\right|\right\}$ is not weakly convergent.

EXAMPLE 4.3 If $A \subset c_{o}$ is relatively weakly compact then $\operatorname{sol}(A)$ is relatively weakly compact.

Proof. Since $c_{o}$ is an $A M$-space, the lattice operations are sequentially weakly continuous. In fact, by the Kakutani representation theorem every AM-space is a closed sublattice of some space of the type $C(\Omega)$, where $\Omega$ is compact. But $x_{n} \rightarrow 0$ weakly in $C(\Omega)$ if and only if $\left(x_{n}\right)$ is norm bounded and $x_{n}(t) \rightarrow 0$ for every $t \in \Omega$. Thus, for example $x_{n} \rightarrow 0$ weakly implies $\left|x_{n}\right| \rightarrow 0$ weakly. Therefore the set $|A|=\{|a|: a \in A\}$ is relatively weakly compact. From Lemma 4.1b) $\operatorname{sol}(|A|)$ is relatively weakly compact, but $\operatorname{sol}(|A|)=\operatorname{sol}(A)$ and we are done. Q.E.D.

From Lemma 4.1 and Example 4.3 we have the following theorem which is mainly due to Aliprantis-Burkinshaw ([4]).

THEOREM 4.4 Factorization of weakly compact operators $T: X \rightarrow Y$ through reflexive Banach lattice holds if either:

i) $Y$ is a KB-space;

ii) $Y=c_{o}$;

iii) $T$ is positive and $Y$ has order continuous norm;

iv) $X^{*}$ has order continuous norm;

v) $T$ has a factorization $T=R S$, where $S: X \rightarrow Z$ and $R: Z \rightarrow Y$ are weakly compact and $Z$ is a Banach lattice.

EXAMPLE 4.5 (Counterexample; Talagrand, [29]) There is a weakly compact positive operator $T: \ell_{1} \rightarrow C([0,1])$ for which factorization through a reflexive Banach lattice does not hold.

In the proofs of the factorization Theorem 4.4iv) and v) it was important to know when the following property holds:

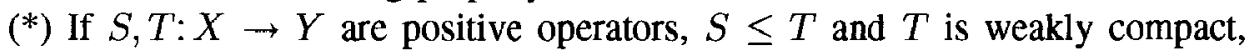
then $S$ is weakly compact.

The answer was given by Aliprantis-Burkinshaw ([3]) and Wickstead ([30]).

THEOREM 4.6 a) Let $X$ be a Banach lattice and let $T: X \rightarrow X$ be a positive weakly compact operator. If an operator $S: X \rightarrow X$ satisfies $0 \leq S \leq T$, then $S^{2}$ is a weakly compact operator.

b) Property (*) holds if and only if either $X^{*}$ or $Y$ has an order continuous norm. 


\section{Interpolation of Weakly Compact Operators}

Let $\bar{X}=\left(X_{o}, X_{1}\right)$ be a Banach pair, i.e., $X_{o}$ and $X_{1}$ are Banach spaces continuously imbedded into a Hausdorff topological vector space. Define, as usual the spaces $\Delta(\bar{X})=X_{o} \cap X_{1}$ and $\Sigma(\bar{X})=X_{o}+X_{1}$ with the norms $\|x\|_{\Delta}=\max \left(\|x\|_{X_{o}},\|x\|_{X_{1}}\right)$ and $\|x\|_{\Sigma}=K(1, x)$, where for $t>0$,

$$
K(t, x)=\inf \left\{\left\|x_{o}\right\|_{X_{o}}+t\left\|x_{1}\right\|_{X_{1}}: x=x_{o}+x_{1}, x_{o} \in X_{o}, x_{1} \in X_{1}\right\} .
$$

For $0<\theta<1$ and $1 \leq p \leq \infty$, the Lions-Peetre interpolation space $\bar{X}_{\theta, p}=$ $\left(X_{o}, X_{1}\right)_{\theta, p}$ is the space of all $x \in \Sigma(\bar{X})$ for which the norm

$$
\|x\|_{\theta, p}=\left(\sum_{n \in \mathbf{Z}}\left(2^{-\theta n} K\left(2^{n}, x\right)\right)^{p}\right)^{\frac{1}{p}}
$$

is finite.

Let us note that $\mu_{n}(x) \leq K\left(2^{n}, x\right) \leq 2 \mu_{n}(x)$, where $\mu_{n}$ is the Minkowski functional of the set $V_{n}=B_{X_{o}}+2^{-n} B_{X_{1}}\left(B_{X_{i}}\right.$ is the unit ball of $\left.X_{i}, i=0,1\right)$, i.e., $\mu_{n}(x)=\inf \left\{\alpha>0: x \in \alpha V_{n}\right\}$. Hence $\bar{X}_{\theta, p}$ consists of those $x \in \Sigma(\bar{X})$ for which the norm

$$
\|x\|_{\theta, p}^{1}=\left(\sum_{n \in \mathbf{Z}}\left(2^{-\theta n} \mu_{n}(x)\right)^{p}\right)^{\frac{1}{p}}
$$

is finite. If in the above definitions the sums are only over natural $n$, then we have the definition of the space $\bar{X}_{\theta, p}^{+}$. Always $\bar{X}_{\theta, p} \subset \bar{X}_{\theta, p}^{+}$; if $X_{o} \subset X_{1}$, then $\bar{X}_{\theta, p}=\bar{X}_{\theta, p}^{+}$.

Now let $\bar{X}=\left(X_{o}, X_{1}\right)$ and $\bar{Y}=\left(Y_{o}, Y_{1}\right)$ be two Banach pairs. We say that a linear operator $T$ is bounded from the pair $\bar{X}$ into the pair $\bar{Y}$, and write $T: \bar{X} \rightarrow \bar{Y}$, if $T: \Sigma(\bar{X}) \rightarrow \Sigma(\bar{Y})$ is a bounded linear operator and the restriction of $T$ to the space $X_{i}$ is a bounded operator from $X_{i}$ into $Y_{i}, i=0,1$.

From the construction of the Lions-Peetre spaces we have that if $T: \bar{X} \rightarrow \bar{Y}$, then $T: \bar{X}_{\theta, p} \rightarrow \bar{Y}_{\theta, p}$ is bounded and

$$
\|T\|_{\bar{X}_{\theta, p} \rightarrow \bar{Y}_{\theta, p}} \leq\|T\|_{X_{o} \rightarrow Y_{o}}^{1-\theta}\|T\|_{X_{1} \rightarrow Y_{1}}^{\theta} .
$$

The following result can be regarded as a generalization of the theorem of Beauzamy [6] about the reflexivity of $\bar{X}_{\theta, p}$.

THEOREM 5.1 (Maligranda-Quevedo, [20]) Let $1<p<\infty$ and $T: \bar{X} \rightarrow \bar{Y}$. Then $T: \bar{X}_{\theta, p} \rightarrow \bar{Y}_{\theta, p}$ is weakly compact if and only if $T: \Delta(\bar{X}) \rightarrow \Sigma(\bar{Y})$ is weakly compact.

LEMMA $5.2(\mathrm{M}-\mathrm{Q},[20])$ a) Let $T: \Sigma(\bar{X}) \rightarrow Y$ be a continuous operator. Then the following are equivalent: 
i) $T: \Delta(\bar{X}) \rightarrow Y$ is weakly compact;

ii) $T:\left(\Delta(\bar{X}, \Sigma(\bar{X}))_{\theta, p} \rightarrow Y\right.$ is weakly compact, for all $0<\theta<1$ and all $1<p<\infty$

iii) $T: \bar{X}_{\theta, p} \rightarrow Y$ is weakly compact, for all $0<\theta<1$ and all $1<p<\infty$. b) Let $1<p<\infty$ and let $T: X \rightarrow \bar{Y}_{\theta, p}$ be a continuous operator. Then $T$ is weakly compact if and only if $T: X \rightarrow \Sigma(\bar{Y})$ is weakly compact.

Proof. a) i) $\Rightarrow$ ii) The interpolation space

$$
Z=(\Delta(\bar{X}), \Sigma(\bar{X}))_{\theta, p}
$$

is a DFJP space constructed from $X=\Sigma(\bar{X})$ and $W=I\left(B_{\Delta(\bar{X})}\right)$, where $I$ is the inclusion $I: \Delta(\bar{X}) \rightarrow \Sigma(\bar{X})$. Since $T(W)$ is relatively weakly compact in $Y$, it is possible to prove that $T \circ J: X \rightarrow Y$ is weakly compact.

ii) $\Rightarrow$ iii). The interpolation space $\bar{X}_{\theta, p}$ is not, in general, a DFJP space. But is proved in [19] that it is imbeddable into $Z=(\Delta(\bar{X}), \Sigma(\bar{X}))_{\theta, p}$, i.e., if $\frac{1}{2} \leq \theta<1$, then

$$
Z=(\Sigma(\bar{X}), \Delta(\bar{X}))_{1-\theta, p}=\bar{X}_{\theta, p}+\bar{X}_{1-\theta, p}
$$

and so $\bar{X}_{\theta, p}$ and $\bar{X}_{1-\theta, p}$ are in $Z$.

That iii) implies i) is obvious, because $\bar{X}_{\theta, p} \supset \Delta(\bar{X})$.

b) The proof of this part follows from the fact that if $1<p<\infty$, then the imbedding $J: \bar{Y}_{\theta, p} \rightarrow \Sigma(\bar{Y})$ is a Tauberian operator, and from the characterization of Tauberian operators by relatively weakly compact sets given by Kalton and Wilansky (cf. [15, Thm. 3.2] and [24, Thm. 1.4]). Q.E.D.

Let us note that Lemma 5.2a) is also true for compact, weakly precompact (=Rosenthal) and Banach-Saks operators. Moreover, the equivalence between i) and ii) is true even for surjective closed operator ideals (see [14, Prop. 1.7]). Lemma $5.2 \mathrm{~b}$ ) and Theorem 5.1 are also true for weakly precompact operators. Theorem 5.1 holds for such classes of operators which are preserved by Lemma 5.2a) and Tauberian operators.

If in our Theorem 5.1 the operator is taken to be the identity, then we have the result of Beauzamy.

COROLLARY 5.3 (Beauzamy, [6]) Let $0<\theta<1$ and $1<p<\infty$. The space $\bar{X}_{\theta, p}$ is reflexive if and only if the pair $\bar{X}$ is weakly compact, i.e., the imbedding $I: \Delta(\bar{X}) \rightarrow \Sigma(\bar{X})$ is weakly compact.

Another proof of this result is the following.

If the imbedding $I$ is weakly compact, then the DFJP space $(\Delta(\bar{X}), \Sigma(\bar{X}))_{\frac{1}{2}, p}$ is reflexive. But from [19] we have that $(\Delta(\bar{X}), \Sigma(\bar{X}))_{\frac{1}{2}, p}=\bar{X}_{\frac{1}{2}, p}$ and from the reiteration theorem from the complex interpolation space (see [7, Thm. 4.7.2]):

$$
\bar{X}_{\theta, p}=\left[\bar{X}_{\theta_{\circ}, p}, \bar{X}_{\frac{1}{2}, p}\right]_{\theta_{1}}
$$


for some $\theta_{\circ}$ and $\theta_{1}$ in $(0,1)$. Now, using the above and Calderón's result (if either $X_{\circ}$ or $X_{1}$ is a reflexive space, then the complex interpolation space $\left[X_{0}, X_{1}\right]_{\theta}$ is also reflexive, see $\left[9\right.$, Thm. 12.2]) we have that $\bar{X}_{\theta, p}$ is reflexive.

Let us note that in the nontrivial case, i.e., when the imbedding $I$ is nonclosed, the space $\bar{X}_{\theta, 1}$ is nonreflexive. Indeed, by Levy's [17] result it contains an isomorphic copy of $\ell^{1}$. However, $\bar{X}_{\theta, 1}$ is reflexive if and only if $I$ is weakly compact and closed, which means that $\bar{X}_{\theta, 1}=\Delta(\bar{X})$ and $\Delta(\bar{X})$ is reflexive (cf. [27]).

There are simple counterexamples showing that Theorem 5.1 (even Corollary 5.3) is not true for the complex method of interpolation.

EXAMPLE 5.4 (Maligranda, [19]) For $1<p<\infty$ let us consider the Marcinkiewicz space

$$
L^{p \infty}=L^{p \infty}(0,1)=\left(L^{1}(0,1), L^{\infty}(0,1)\right)_{\frac{1}{p}, \infty}
$$

and the closed subspace $L_{\circ}^{p \infty}$ which is the closure of $L^{\infty}$ in $L^{p \infty}$. For $0<\theta<1$ and $1<p<q<\infty$, let $\frac{1}{r}=(1-\theta) / p+\theta / q$. Then the pair $\left\{L_{\circ}^{p \infty}, L_{\circ}^{q \infty}\right\}$ is weakly compact because $L_{\circ}^{q \infty} \subset L^{r} \subset L_{\circ}^{p \infty}$ and $\left[L_{\circ}^{p \infty}, L_{\circ}^{q \infty}\right]_{\theta}=L_{\circ}^{r \infty}$ are not reflexive spaces.

EXAMPLE 5.5 Denote by $F L_{1}$ the Fourier transforms of functions in $L^{1}(0,2 \pi)$, i.e., the space of sequences $\left(a_{n}\right)_{-\infty}^{\infty}$ such that $\sum_{-\infty}^{\infty} a_{n} e^{i n x} \in L^{1}(0,2 \pi)$ with the norm

$$
\left\|\left\{a_{n}\right\}\right\|_{F L_{1}}=\left\|\sum_{-\infty}^{\infty} a_{n} e^{i n x}\right\|_{L_{1}} .
$$

The pair $\overline{F L_{1}^{\theta}}=\left\{F L_{1}\left(2^{\theta n}\right), F L_{1}\left(2^{(\theta-1) n}\right)\right\}$ is weakly compact because $\ell_{2}$ is an interpolation space (more precisely, the Gustavsson-Peetre or Ovchinnikov method applied to this pair gives $\ell_{2}$, see [26]). Moreover, the complex method for this pair gives $\left[F L_{1}\left(2^{\theta n}\right), F L_{1}\left(2^{(\theta-1) n}\right)\right]_{\theta}=F L_{1}$ (see [26]) and the space $F L_{1}$ is not reflexive. The techniques used in Theorem 5.1 also work for the more general real method of interpolation (cf. [21], [27]). It is also possible to formulate and prove a theorem for arbitrary interpolation functors ("abstract nonsense').

THEOREM 5.6 Let $T: \bar{X} \rightarrow \bar{Y}$ be such that $T: \Delta(\bar{X}) \rightarrow \Sigma(\bar{Y})$ is weakly compact. Assume that $F$ is an interpolation functor such that:

i) for every $\varepsilon>0$ there exists $t>0$ such that

$$
B_{F(\bar{X})} \subset t B_{\Delta(\bar{X})}+\varepsilon B_{\Sigma(\bar{X})}
$$

ii) the imbedding $J: F(\bar{Y}) \rightarrow \Sigma(\bar{Y})$ is a Tauberian operator.

Then $T: F(\bar{X}) \rightarrow F(\bar{Y})$ is weakly compact. 


\section{References}

1. Yu. A. Abramovich, Weakly compact sets in topological $K$-spaces, Teor. Funkcii Funkcional. Anal. i Prilozen. 15 (1972), pp. 27-35 (in Russian).

2. M. H. Aizenshtein, Duality of interpolation functors, Studies in the Theory of Functions of Several Variables Yaroslavl (1986), pp. 3-11 (in Russian).

3. C. D. Aliprantis, 0. Burkinshaw, On weakly compact operators on Banach lattices, Proc. Amer. Math. Soc. 83 (1981), pp. 573-578.

4. C. D. Aliprantis, 0. Burkinshaw, Factoring compact and weakly compact operators through reflexive Banach lattices, Trans. Amer. Math. Soc. 283 (1984), pp. 369-381.

5. C. D. Aliprantis, 0. Burkinshaw, Positive Operators, Acad. Press, New York, 1985.

6. B. Beauzamy, Espaces d'Interpolation Reels: Topologie et Géometrie, Lecture Notes in Math. 666, Springer-Verlag, Berlin-Heidelberg-New York, 1976.

7. J. Bergh, J. Lofstrom, Interpolation Spaces. An introduction, Springer-Verlag, BerlinHeidelberg-New York, 1976.

8. Yu. A. Brudnyi, N. YA. Krugljak, Interpolation Functors and Interpolation Spaces I North-Holland, Amsterdam (to appear).

9. A. P. Calderon, Intermediate spaces and interpolation, the complex method, Studia Math. 24 (1964), pp. 133-190.

10. M. Cwikel, Real and complex interpolation and extrapolation of compact operators, Haifa 1990 (manuscript).

11. W.J. Davies, T. Figiel, W.B. Johnson, A. Pelczynski, Factoring weakly compact operators, J. Functional Anal. 17 (1974), pp. 311-327.

12. J. Diestel, Sequences and Series in Banach Spaces, Springer-Verlag, Berlin-HeidelbergNew York, 1984.

13. K. Floret, Weakly Compact Sets, Lecture Notes in Math. 801, Springer-Verlag, BerlinHeidelberg-New York, 1980.

14. B. Heinrich, Closed operator ideals and interpolation, J. Functional Anal. 35 (1980), pp. 397-411.

15. N.J. Kalton, A. Wilansky, Tauberian operators on Banach spaces, Proc. Amer. Math. Soc. 57 (1976), pp. 251-255.

16. S.G. Krein, Yu. I. Petunin, E.M. Semenov, Interpolation of Linear Operators, Nauka, Moscow, 1978; English transl.: AMS, Providence, 1982.

17. M. Levy, L'espace d'interpolation réel $\left(A_{\circ}, A_{1}\right)_{\theta, p}$ contient $\ell_{p}$, C.R. Acad. Sci. Paris 289 (1979), pp. 675-677.

18. J. Lindenstrauss, Weakly compact sets - their topological properties and the Banach spaces they generate, in: Proc. Symp. on Infinite Dimensional Topology, Annals of Math. Studies 69 ,Princeton Univ. Press, 1972, pp. 235-273.

19. L. Maligranda, Interpolation between sum and intersection of Banach spaces, J. Approx. Theory 47 (1986), pp. 42-53.

20. L. Maligranda, A. Quevedo, Interpolation of weakly compact operators, Arch. Math. 55 (1990), pp. 280-284.

21. M. Mastylo, On interpolation of weakly compact operators, Hokkaido Math. J. (to appear (1991?)).

22. P. Meyer-Nieberg, Zur schwachen kompaktheit in Banachverbänden, Math. Z. 134 (1973), pp. 303-315.

23. R.D. Neidinger, Properties of Tauberian operators on Banach spaces, Ph.D. Dissertation, Univ. of Texas at Austin (1984), 144 pp.

24. R.D. Neidinger, Factoring operators through hereditarily- $\ell_{p}$ spaces, Lecture Notes in Math. 1166, Springer-Verlag, Berlin-Heidelberg-New York, 1985, pp. 116-128.

25. R.D. Neidinger, Concepts in the real interpolation of Banach spaces, Longhorn Notes, The Univ. of Texas at Austin, Functional Analysis Seminar 1986-87 pp. 1-15.

26. V.I. Ovchinnikov, The Method of Orbits in Interpolation, Math. Reports 1 Part 2, Harwood Academic Publishers, 1984, 349-516.

27. A. Quevedo, Some remarks on the reflexivity of the real interpolation spaces, J. Math. Anal. Appl. (to appear). 
28. J.V. Ryff, The weak closure of a certain set in $\ell^{1}$, Amer. Math. Monthly 81 (1974), pp. 69-70.

29. M. Talagrand, Some weakly compact operators between Banach lattices do not factor through reflexive Banach lattices, Proc. Amer. Math. Soc. 96 (1986), pp. 95-102.

30. A.W. Wickstead, Extremal structure of cones of operators, Quart. J. Math. Oxford Ser. (2) 32 (1981), pp. 239-253. 